\title{
TIEMPO Y MEMORIA DEL INSTITUTO-ESCUELA
}

\section{TIME AND MEMORY OF THE INSTITUTO-ESCUELA}

http://dx.doi.org/10.15304/ie.28.5482

\author{
Encarnación Martínez Alfaro \\ Instituto Isabel la Católica (Madrid) \\ encarnacion.alf@gmail.com
}

\section{RESUMEN}

La conmemoración del centenario del Instituto-Escuela en 2018 es una oportunidad para reivindicar una institución trascendental en la educación española y, sin embargo, bastante desconocida al margen de determinados ámbitos académicos. Quizás es la menos conocida de las instituciones que dirigió la Junta para Ampliación de Estudios e Investigaciones Científicas (JAE) con el fin de modernizar e impulsar la ciencia, la cultura y la educación españolas, en el primer tercio del siglo XX. El InstitutoEscuela fue concebido como un centro experimental de Enseñanza Secundaria y de formación del profesorado, donde se ensayó un nuevo y ambicioso modo de educar que recogía la herencia de la Institución Libre de Enseñanza (ILE) y la pedagogía europea más avanzada de la época. Abrió sus puertas en 1918 en Madrid y desarrolló su proyecto pedagógico sin interrupción hasta 1936, cuando estalló la Guerra Civil. Fue tras ésta cuando su historia y su legado entraron en un largo periodo de casi absoluto olvido hasta fechas bastante recientes.

Una de las sedes que tuvo el Instituto-Escuela, la Sección Retiro, localizada junto al famoso parque madrileño, es desde 1939 el Instituto Isabel la Católica. Por este motivo en él se han conservado, además del propio edificio, un archivo con expedientes de alumnos, un fondo bibliográfico de unos 1.500 libros con el sello del propio centro y abundante material científico-didáctico. Desde el año 2006, un grupo de profesores hemos venido trabajando en la recuperación del patrimonio histórico-educativo del Instituto-Escuela para dar a conocer su existencia y significado en la historia de la educación española, descubrir la vigencia de muchas de las innovaciones pedagógicas que introdujo, y utilizarlo como recurso didáctico en proyectos interdisciplinares que llevamos a cabo cada curso con nuestros alumnos. Palabras clave: Instituto-Escuela, innovaciones pedagógicas, patrimonio histórico-educativo, recurso didáctico.

\begin{abstract}
The commemoration of the centenary of the Instituto-Escuela in 2018 is an opportunity to vindicate a transcendental institution in Spanish education and, however, quite unknown, outside certain academic areas. Perhaps it is the least known of the institutions that led the Junta para Ampliación de Estudios e Investigaciones Cientificas (JAE) in order to modernize and promote Spanish science, culture and education, in the first third of the 20th century. The Instituto-Escuela was conceived as an experimental center for Secondary Education and teacher training, where a new and ambitious way of educating was
\end{abstract}

Recibido: 23/IX/2018. Aceptado: X/2018 
tried which gathered the legacy of the Institución Libre de Enseñanza (ILE) and the most advanced European pedagogy of the time. It opened its doors in 1918 in Madrid and developed its pedagogical project without interruption until 1936, when the Civil War broke out. It was after this when his history and his legacy entered a long period of almost absolute oblivion until fairly recent dates.

One of the seats that the Instituto-Escuela had, the Retiro Section, located next to Madrid's famous park, is since 1939 the Instituto Isabel la Católica. For this reason it has kept, in addition to the building itself, a file with records of students, a library of about 1,500 books with the seal of the center itself and abundant scientific-educational material. Since 2006, a group of teachers have been working on the recovery of the historical-educational heritage of the Instituto-Escuela to make known its existence and meaning in the history of Spanish education, discover the validity of many of the pedagogical innovations that introduced, and use it as a didactic resource in interdisciplinary projects that we carry out each course with our students.

Keywords: Instituto-Escuela, pedagogical innovations, historical-educational heritage, teaching resource.

\section{INTRODUCCIÓN}

A principios del siglo XX la Enseñanza Secundaria española tenía graves carencias que hacían inaplazable una revisión en profundidad. En la exposición del Real Decreto del 12 de abril de 1901 sobre la reforma de la enseñanza oficial, siendo ministro de Instrucción Pública el conde de Romanones, en pleno debate regeneracionista tras la crisis del 98, se hace referencia a la situación de postración en que se hallaba:

La enseñanza oficial, [...] atraviesa un periodo angustioso y se ve abandonada por la confianza de las familias, como demuestran las estadísticas, pues el número de alumnos de la enseñanza oficial no llega actualmente al 25 por 100 de la población escolar no oficial. (Utande, 1964, p. 375).

Romanones se plantea la renovación de la enseñanza secundaria, como se había hecho en otros países europeos, para adaptarla a las necesidades educativas del nuestro.

Trátase tan sólo de organizar la enseñanza de modo que responda a un estado social tan complejo como el presente, y a unas necesidades tan variadas como las de la moderna vida comercial, industrial y científica. (Utande, 1964, p. 389).

Pero Romanones es consciente de los obstáculos existentes para sacar adelante su reforma de la enseñanza, como la exigüidad de los presupuestos, las aulas excesivamente masificadas y las dificultades para reorganizar las Escuelas Normales de Maestros. El citado decreto hace también referencia a dos cuestiones polémicas del momento que después se planteará también el InstitutoEscuela: los exámenes y los libros de texto.

A la vista de la situación, los ministros liberales que sucedieron a Romanones tomaron medidas encaminadas a potenciar los institutos Generales y Técnicos de Segunda Enseñanza (López-Ocón, 2014), renovaron los edificios de muchos institutos y mejoraron su dotación económica, en especial la destinada a la compra de material científico y didáctico con el fin de promover la enseñanza 
experimental. También incrementaron las plantillas de profesores, que en algunos casos impartían sus clases con métodos pedagógicos más modernos.

\section{EL INSTITUTO-ESCUELA, UNA EXPERIENCIA EDUCATIVA SINGULAR}

Sin embargo, pese a las medidas positivas tomadas por la administración educativa y a la sensibilidad modernizadora de algunos profesores, las carencias de la Enseñanza Secundaria española en 1918 seguían sin resolverse. Para solucionar esas carencias, a iniciativa del ministro de Instrucción Pública, Santiago Alba, se creó el Instituto-Escuela, que nació como un centro experimental donde ensayar la reforma educativa y formar nuevos profesores. El Instituto-Escuela debía disponer de dos requisitos fundamentales: libertad de acción y continuidad en el tiempo para observar sus resultados (Díaz de Laguardia, 1988, p. 314). Por este motivo, para evitar que el ensayo estuviera sometido a los vaivenes de la política, el Instituto-Escuela se puso bajo la dirección de la Junta para Ampliación de Estudios e Investigaciones Científicas (JAE)ํ.

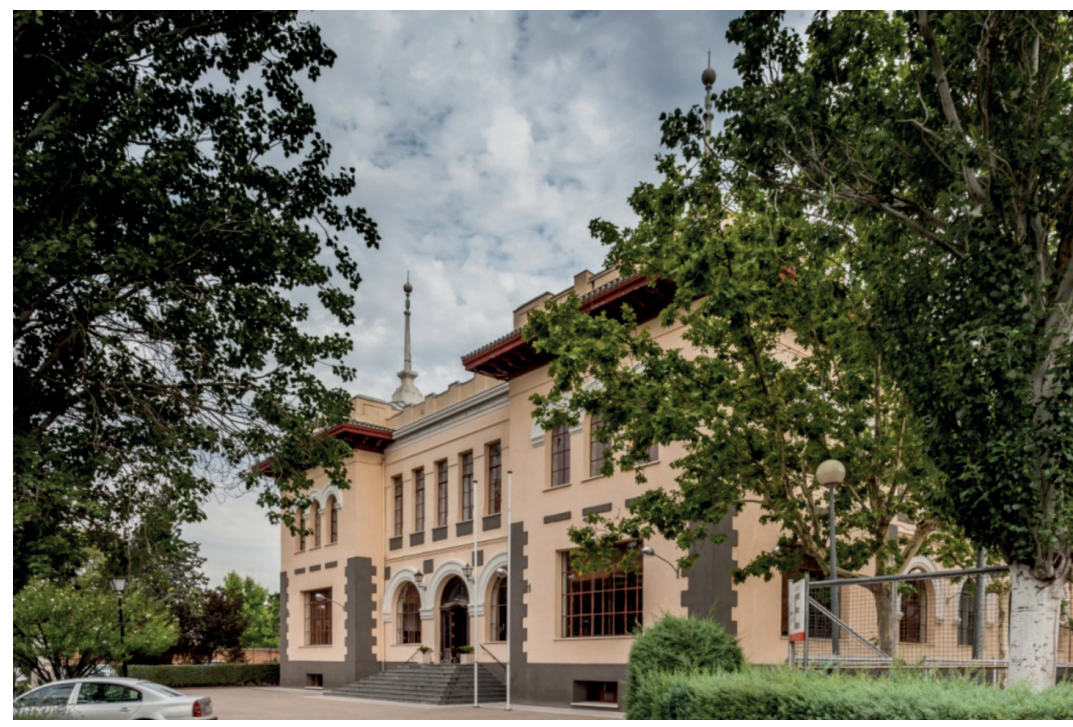

Figura 1. Sección Retiro del Instituto-Escuela. Actual Instituto Isabel la Católica.

Las bases de su proyecto fueron la Institución Libre de Enseñanza y la pedagogía implantada en los países europeos más avanzados. Por eso muchos profesores del Instituto-Escuela estuvieron pensionados por la JAE en Francia, Alemania e Inglaterra, principalmente, para conocer los métodos pedagógicos de sus centros educativos más modernos y luego aplicarlos en sus clases.

José Castillejo, secretario de la JAE, fue quien ejerció en los primeros años la dirección efectiva del Instituto-Escuela. 
El funcionamiento del Instituto-Escuela fue como el de un laboratorio más de la JAE, de manera que su práctica pedagógica era revisada periódicamente para comprobar su idoneidad. Sus resultados se evaluaban y, cuando era necesario, se corregía, contando en algunas ocasiones con la opinión de los padres de los alumnos, quienes también conocían los progresos o dificultades de aprendizaje de sus hijos ${ }^{2}$.

El experimento educativo del Instituto-Escuela se basó en tres elementos fundamentales: un plan de estudios propio, una pedagogía innovadora y un equipo de profesores que trabajó de forma coordinada.

\section{EL PLAN DE ESTUDIOS}

El Instituto-Escuela diseñó un plan de estudios propio para el Bachillerato que abarcaba seis cursos. El alumno, al llegar al quinto curso, pensando en sus futuros estudios universitarios, podía optar por el Bachillerato de Ciencias o el de Letras. Para garantizar la formación del alumnado, el Instituto-Escuela contemplaba un amplio abanico de medidas innovadoras; entre ellas, una reducción significativa del número de alumnos por aula (hasta 30), la instalación de laboratorios como espacios para una verdadera formación científica y experimental, el incremento del número de horas de las materias (en particular en las de ciencias y en los idiomas) y la realización de excursiones para el estudio directo de las cosas. En el aprendizaje de los idiomas (francés, inglés y alemán), desempeñaron un papel muy importante los viajes al extranjero y los intercambios con centros europeos por su novedad y eficacia pedagógica. Para atender a la formación integral del alumno, el Instituto-Escuela introdujo en su plan de estudios enseñanzas que contribuían a su desarrollo físico, formación estética y destrezas manuales, con poca o ninguna tradición en España, como los Juegos (o Deportes), el Dibujo, la Música y los Trabajos Manuales.

Otro de los aspectos fundamentales que abordó el plan de estudios del Instituto-Escuela fue el de implantar la enseñanza cíclica. Con ello pretendían que las materias de Bachillerato tuvieran la necesaria continuidad y coherencia, profundizando en sus contenidos progresivamente y evitando que quedasen como estudios sueltos e inconexos (Martínez Alfaro, 2009, p. 88).

Esa misma continuidad y coherencia también se pretendió dar entre las enseñanzas de la Primaria y el Bachillerato. Por esta razón el Instituto-Escuela tuvo una Sección Preparatoria, para los alumnos de 8 a 10 años. La continuidad entre ambas etapas se buscó a través de la aplicación de los mismos principios pedagógicos (inspirados en gran medida por María de Maeztu, directora de la Sección Preparatoria), y la coherencia de los contenidos relacionados en las programaciones de las materias. Pero, en la práctica, este objetivo fue difícil de cumplir. Como señala el informe de la Junta sobre los seis primeros años de funcionamiento del Instituto-Escuela, "la tradicional separación y mutua incomprensión entre los maestros primarios y los catedráticos de Instituto" (JAE, 1925, p. 375), junto a otros motivos, como el excesivo trabajo de los profesores y la falta de un local único, explican que el cumplimiento de ese objetivo de continuidad no se lograra plenamente.

2 Además de la comunicación periódica con las familias, el Instituto-Escuela hizo dos consultas generales a los padres, durante los cursos 1921-1922 y 1923-1924, para que comunicasen sus apreciaciones y sugerencias. (Martínez Alfaro, 2009, p. 301). 


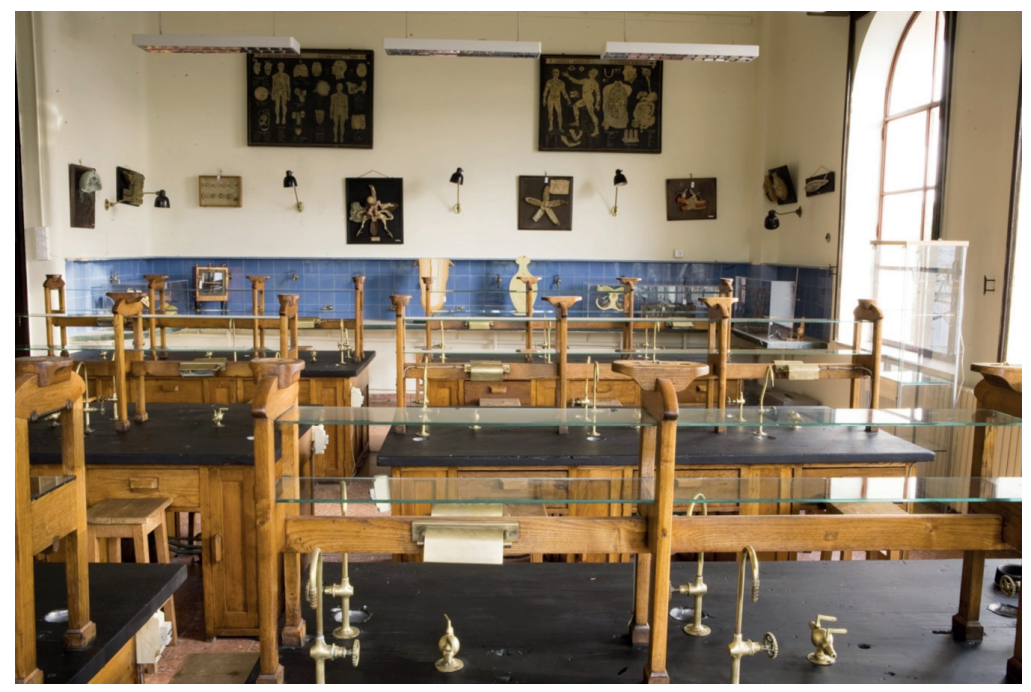

Figura 2. Laboratorio de Biología del Instituto-Escuela. Sección Retiro.

\section{LA METODOLOGÍA}

El Instituto-Escuela basó su método de enseñanza en el diálogo continuo entre el alumno y el profesor, la observación directa de las cosas, la comprensión, la reflexión, el razonamiento y la experimentación como elementos clave de un aprendizaje autónomo. En relación con esta metodología, las novedades pedagógicas más conocidas introducidas por el Instituto-Escuela fueron la supresión de los exámenes y la eliminación de los libros de texto. Ambas cuestiones estaban planteadas desde principios de siglo, como se ha comentado anteriormente, y requieren algunas matizaciones.

La supresión de los exámenes significaba para el Instituto-Escuela descartar la práctica del examen único de fin de curso para aprobar una materia, como estaba establecido en los institutos oficiales. Con un único examen sólo se podían valorar los conocimientos memorísticos que el alumno hubiera adquirido, pero difícilmente su madurez intelectual y sus capacidades; estudiaba para aprobar el examen, es decir, sólo memorizaba contenidos, a menudo desfasados. E1 Instituto-Escuela estableció otro modelo para valorar los aprendizajes del alumno, anticipando lo que llamamos la evaluación continua. En lugar de calificar al alumno con la nota de una única prueba por materia a fin de curso, se impuso la realización de tres evaluaciones trimestrales ${ }^{3}$, las cuales permitían a los profesores valorar su progreso a partir de las intervenciones en el aula, los ejercicios diarios, los cuadernos de clase, las prácticas de laboratorio y las pruebas periódicas orales o escritas (Martínez Alfaro, 2009, p. 277).

3 Las hojas de evaluación trimestrales por curso de cada alumno, en los expedientes de los alumnos del Instituto-Escuela conservados en el Instituto Isabel la Católica, dejan constancia de esta práctica. 


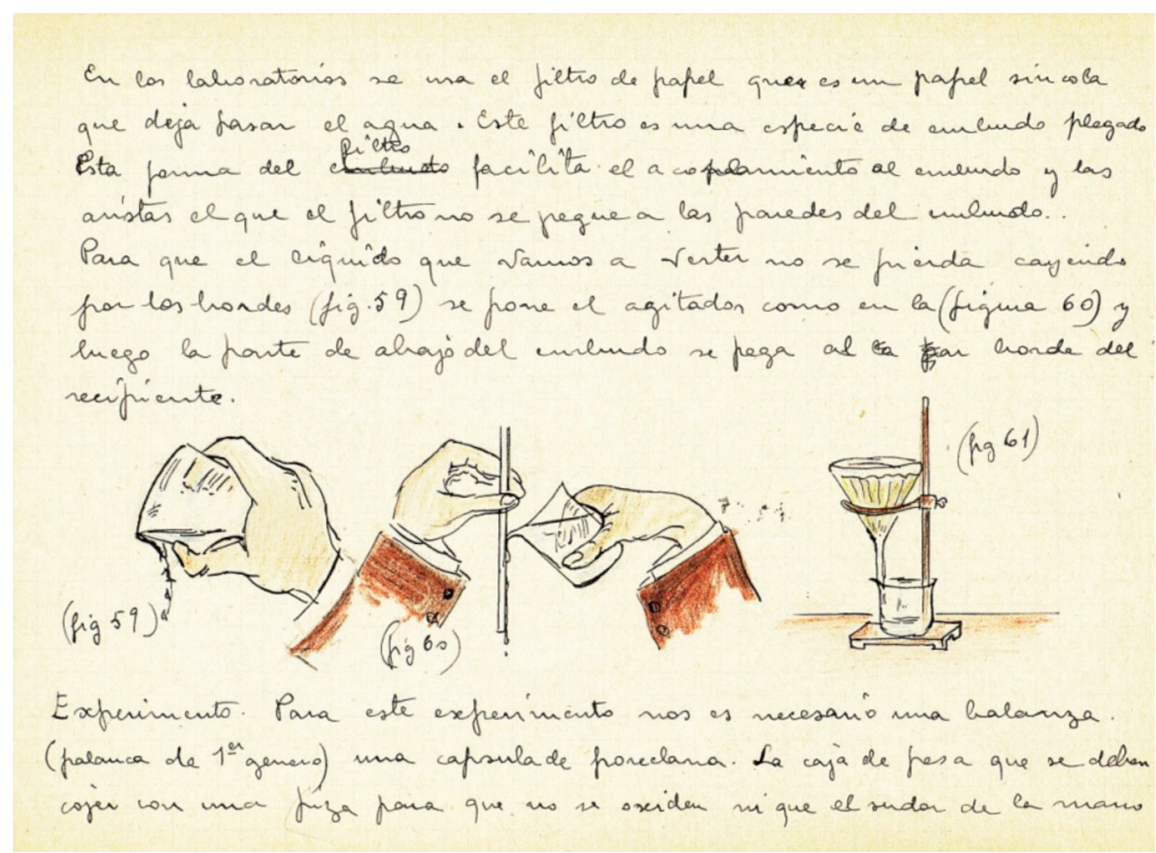

Figura 3. Cuaderno de Física y Química del alumno del Instituto-Escuela José Subirana en $2^{\circ}$ de Bachillerato. Curso 1923-1924. Archivo Instituto Isabel la Católica.

Respecto a los libros de texto, la polémica sobre su uso se remonta también a finales del siglo XIX y principios del XX, y aparece igualmente reflejada en el Plan Romanones, donde se criticaba que los libros de texto "no se escriben en la generalidad de los casos con propósito verdaderamente didáctico, sino para dar contestación a las preguntas en los exámenes” (Utande, 1964, pp. 378-379). Los libros de texto de la época, lejos de formar al alumno, sólo servían para aprendizajes eminentemente memorísticos cuyo fin no era otro que salvar el escollo del examen de fin de curso. Además se les acusaba de responder a los intereses económicos de los catedráticos, pues los escribían para completar su sueldo (Canes Garrido, 2001, p. 377). El Instituto-Escuela prescindió de los libros de texto y los sustituyó por libros de estudio y de consulta y, sobre todo, por los cuadernos de clase. Los cuadernos, junto con los trabajos escolares, propiciaban, según María de Maeztu, una mayor autonomía en el pensamiento y el estudio (JAE, 1925, p. 34), ya que el alumno debía reelaborar y anotar en ellos los contenidos tratados en el aula, en los laboratorios, en las visitas a los museos y en las excursiones que hacían a lugares de interés formativo, como el campo, las fábricas o las ciudades con patrimonio histórico-artístico (Martínez Alfaro y Masip, 2012b, p. 226). Dadas sus ventajas sobre los antipedagógicos libros de texto convencionales, en los primeros cursos de Bachillerato el Instituto-Escuela utilizó preferentemente los cuadernos, lo que no excluía para un aprendizaje más completo la consulta de libros de estudio, como los que aparecen reseñados en las programaciones de las materias elaboradas por los catedráticos del Instituto-Escuela. En los últimos 
cursos de Bachillerato, la Junta recomendaba que se utilizaran libros de estudio y lectura en francés, inglés y alemán (JAE, 1925, p. 397) para ampliar los conocimientos de determinadas materias y mejorar el uso de estas lenguas.

En definitiva, el sistema de enseñanza del Instituto-Escuela aspiraba a una formación más comprensiva, reflexiva y autónoma, de manera que el alumno aprendiera actuando (Palacios Bañuelos, 1988, p. 241). Quizás la mejor prueba de ese método de aprendizaje la encontremos en los experimentos que hacía en los laboratorios. Lo que se buscaba en última instancia era que el alumno participase de manera activa en el descubrimiento de las cosas que eran objeto de estudio y se responsabilizase de su aprendizaje.

\section{LOS PROFESORES Y ALUMNOS DEL INSTITUTO-ESCUELA}

Los profesores que asumieron el proyecto del Instituto-Escuela pertenecían al cuerpo de catedráticos de Enseñanza Secundaria y habían sido seleccionados por la JAE por sus méritos académicos y su identificación con la moderna pedagogía. Su colaboración con las instituciones que dirigía la JAE (Laboratorios científicos, Centro de Estudios Históricos, etc.) hizo que fueran exponentes del nuevo tipo de profesor-científico que estaba actualizado tanto en los contenidos de su materia específica como en los métodos educativos (Casado de Otaola, 2012, p.174). Entre los catedráticos del Instituto-Escuela figuraban científicos de la talla de Miguel A. Catalán o Luis Crespí, y profesores de gran prestigio en sus respectivas especialidades como Samuel Gili Gaya, Manuel de Terán, Jaime Oliver Asín, Andrés León Maroto, Francisco Barnés Salinas o José A. Sánchez Pérez. Algunas de ellos llegaron a ser después académicos, y Francisco Barnés fue Ministro de Instrucción Pública en dos ocasiones durante la II República, en 1933 y 1936. Los catedráticos, además de responsabilizarse de sus tareas docentes, se tuvieron que encargar también de la formación de los llamados profesores aspirantes al Magisterio Secundario.

La formación del profesorado había sido una tarea que la institución Libre de Enseñanza consideraba prioritaria a la hora de afrontar la reforma educativa. Tanto Giner de los Ríos como Cossío habían insistido en la importancia de tener buenos maestros para conseguir el éxito en la tarea educativa, dándole más importancia a este elemento que al material o a las instalaciones escolares (recogido por Ruiz Berrio, 2012, p. 581). Consciente de su importancia, el Instituto-Escuela puso especial empeño en la formación de nuevos profesores. Dirigidos por los catedráticos, los profesores aspirantes al Magisterio Secundario hacían prácticas de dos años en Instituto-Escuela, donde se formaban pedagógicamente, ampliaban los conocimientos de su especialidad en las instituciones dependientes de la JAE y estudiaban los idiomas que iban a facilitarles sus estancias posteriores en el extranjero como pensionados, al mismo tiempo que comenzaban la docencia. Entre los profesores aspirantes formados en el Instituto-Escuela figuran José Camón Aznar, Enrique Lafuente Ferrari, Ángel Valbuena Prat o María Zambrano, que más tarde alcanzaron gran prestigio como catedráticos de universidad, historiadores y ensayistas. 


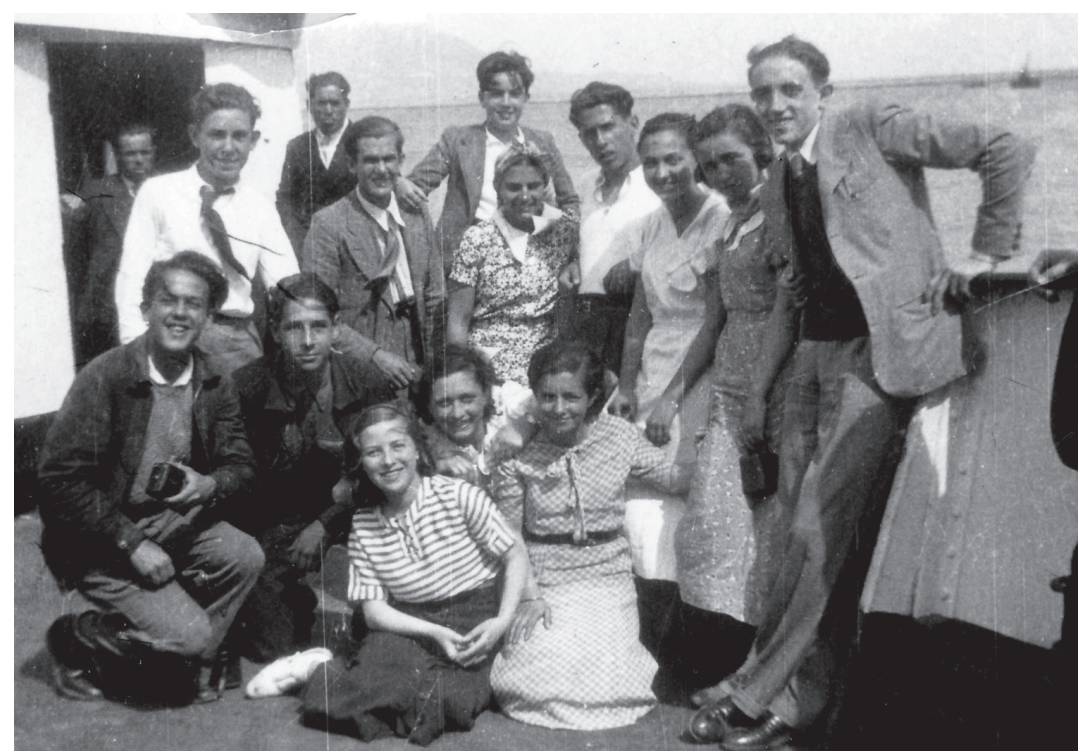

Figura 4. Alumnos del Instituto-Escuela en una excursión a Marruecos en $5^{\circ}$ de Bachillerato.

Curso 1933-1934. Fondo Javier Cabañas.

En el Instituto-Escuela estudiaron los hijos de los científicos e intelectuales que dirigieron los Laboratorios de la JAE y el Centro de Estudios Históricos (CEH) y, en general, los hijos de la burguesía ilustrada (políticos, médicos, abogados, empresarios, artistas, etc.), que simpatizaba con el proyecto educativo del Instituto. La mentalidad liberal y progresista de los padres explica que en el Instituto-Escuela se produjera una situación excepcional en su tiempo, puesto que en él estudiaron casi la misma proporción de alumnas que de alumnos, cuando en los otros institutos madrileños el porcentaje de alumnas en Secundaria no llegaba al veinte por ciento.

Entre los alumnos que estudiaron el Bachillerato en el Instituto-Escuela, cabe mencionar a Julio Caro Baroja, antropólogo, historiador y ensayista; José Ortega Spottorno, fundador del diario El País; Gonzalo Menéndez Pidal, historiador y cineasta; Francisco Bernis Madrazo, cofundador de la Sociedad Española de Ornitología y del Parque de Doñana; y Emilio Garrigues Díaz-Cañabate, diplomático, abogado y escritor. Muchos otros alumnos, la mayoría de ellos científicos y profesores universitarios, como Blas Cabrera Sánchez, Francisco Giral González y Juan Negrín Fidelman, desarrollaron casi sin excepción toda su vida profesional en el exilio tras la Guerra Civil.

De las alumnas ilustres del Instituto-Escuela, hay que recordar a Carmen Bravo-Villasante, escritora; Ángeles Gasset de las Morenas, una de las fundadoras de Colegio-Estudio; Sofía MartínGamero, filóloga; Aurora Villa Olmedo, deportista y oftalmóloga; y Matilde Ucelay Maórtua, primera mujer arquitecta de España. También hubo alumnas que destacaron en el exilio por su labor docente e investigadora, como Carmen de Zulueta Cebrián, profesora en la universidad norteamericana de Harvard. 


\section{LA RECUPERACIÓN DEL PATRIMONIO DEL INSTITUTO-ESCUELA}

En 1939, nada más acabar la Guerra Civil, el experimento reformista del Instituto-Escuela pasó a la historia, la mayor parte de su profesorado fue depurado o tuvo que exiliarse y desde entonces todo su patrimonio sufrió un progresivo abandono. Los edificios de las sedes de Retiro e Hipódromo fueron reconvertidos respectivamente en los nuevos institutos Isabel la Católica y Ramiro de Maeztu. Tanto es así que la memoria del Instituto-Escuela, pasado un tiempo, quedó prácticamente reducida al ámbito exclusivo de los pedagogos y los especialistas en Historia de la Educación.

El descubrimiento casi accidental de unos libros con el sello del Instituto-Escuela en la biblioteca del instituto Isabel la Católica fue el punto de partida para que dos de sus profesoras, quien firma esta colaboración y Carmen Masip Hidalgo, comenzaran a trabajar en el curso 2006-2007 en el estudio y recuperación del valioso patrimonio histórico del Instituto-Escuela (Martínez Alfaro y Masip, 2012a, pp. 135-149). De él se conservaba en nuestro instituto el archivo, gran parte de su diseminada biblioteca y los laboratorios de Física, Química, Biología y Geología con instrumentos científicos y material didáctico. Nuestro trabajo, desde entonces y hasta hoy, ha consistido en la ordenación, catalogación, restauración, conservación, puesta en valor y difusión de ese patrimonio por todos los medios a nuestro alcance. En la catalogación, recibimos el apoyo de instituciones como la Biblioteca Nacional, el Catálogo Colectivo del Patrimonio Bibliográfico y los Museos Nacionales de Ciencias Naturales y Geominero. En las labores de estudio y difusión, fue muy importante nuestra participación en el Programa de I+D CEIMES (Ciencia y Educación en los Institutos Madrileños de Enseñanza Secundaria), auspiciado por la Dirección General de Universidades e Investigación de la Comunidad de Madrid y desarrollado entre 2008 y 2012 (www.ceimes.cchs.csic. es), porque nos permitió establecer contacto con investigadores del CSIC y de algunas universidades madrileñas (UCM, UAM y UNED) interesados en el estudio del patrimonio histórico educativo de los institutos. Para la recuperación y difusión del patrimonio histórico del Instituto-Escuela, también nos han sido especialmente provechosas las Jornadas que con periodicidad anual desde 2007 celebramos en distintos puntos de España los institutos históricos españoles, convertidos en 2010 en Asociación Nacional para la Defensa del Patrimonio de los Institutos Históricos (ANDPIH. http:// asociacioninstitutoshistoricos.org/site/andelpih/index.html). Del mismo modo nos han resultado útiles en la difusión del patrimonio nuestra pertenencia a la Sociedad Española para el Estudio del Patrimonio Histórico Educativo (SEPHE. https://institucional.us.es/paginasephe/) y nuestra inclusión en los programas de la Semana de la Ciencia de la Comunidad de Madrid. Esta labor de difusión se ha complementado desde 2009 con la publicación de diversos estudios y artículos en libros y revistas de educación.

\section{EL PATRIMONIO HISTÓRICO COMO RECURSO DIDÁCTICO}

Con la recuperación casi acabada del patrimonio histórico del Instituto-Escuela, hemos querido restituir su memoria y poner a disposición de los investigadores de la Historia de la Educación un material de gran valor documental, al que se puede acceder en el Instituto Isabel la Católica, donde se conserva. Junto a este posible uso, y habiendo considerado que no puede tratarse 
tampoco como un conjunto de piezas de museo que se exhibe ocasionalmente, nuestro instituto optó por utilizarlo también como un recurso con fines didácticos, es decir, como material de trabajo para profesores y alumnos. Desde el año 2009, un equipo de profesores hemos venido programando y desarrollando una serie de proyectos interdisciplinares de periodicidad anual con grupos de alumnos de $1^{\circ}$ y de $4^{\circ}$ de ESO, con el propósito de que, estudiando el patrimonio, conozcan la historia y el legado del Instituto-Escuela, que es tanto como conocer, entre otras cosas, el origen del centro en el que estudian.

\section{PROYECTOS CON LOS ALUMNOS DE $1^{\circ}$ DE ESO}

Los profesores responsables de los proyectos pertenecen a los departamentos de Ciencias Sociales, Ciencias Naturales, Educación Plástica y Visual y Alemán. Lo que más nos interesa con los alumnos de $1^{\circ}$ de ESO es que conozcan el instituto al que acaban de llegar, mostrarles el patrimonio histórico, explicarles su origen y finalidad educativa, enseñarles a valorarlo y generarles un sentido de pertenencia al centro. Por todo ello, les hacemos una visita guiada a la biblioteca y a los laboratorios históricos, preparada previamente en clase por sus profesores de Ciencias Sociales y Ciencias Naturales. En los laboratorios descubren los materiales científicos, parte de los cuales, como placas de cristal y láminas, dibujan después en la clase de Educación Plástica y Visual. Como el Instituto Isabel la Católica cuenta con una sección bilingüe de alemán y muchos de los objetos científicos del patrimonio son de procedencia alemana (láminas y placas de cristal sobre todo), los profesores de este departamento ayudan a los alumnos a hacer en alemán descripciones muy sencillas de las imágenes científicas que han dibujado y estudiado. La actividad termina cuando la profesora de Educación Plástica y Visual prepara un pequeño cuadernillo con los dibujos comentados de todos los alumnos para que tengan una visión conjunta del trabajo realizado.

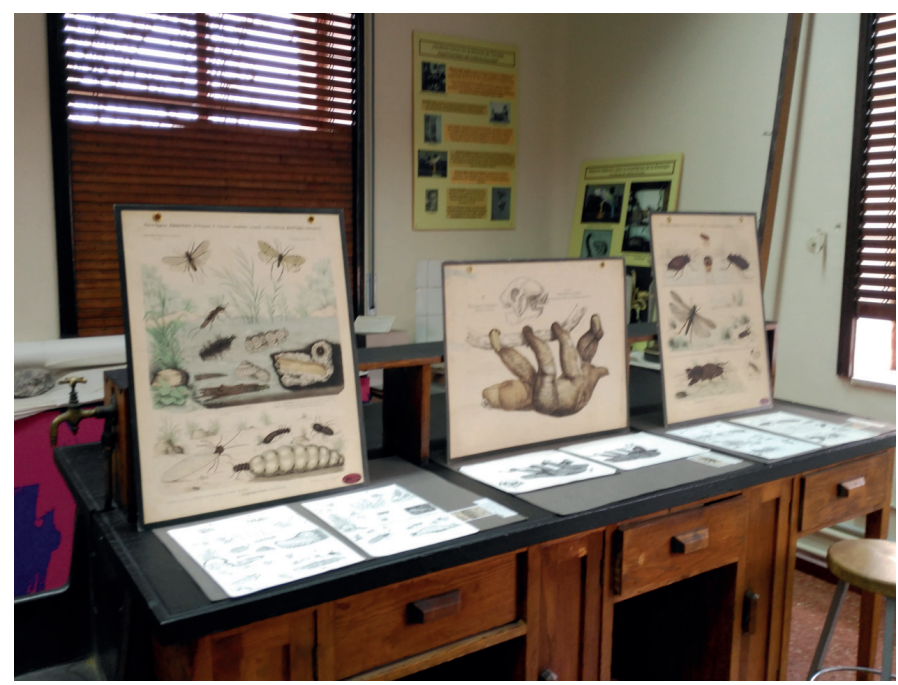

Figura 5. Laminas alemanas del Instituto-Escuela y debajo reproducciones de los alumnos de $1^{\circ}$ de ESO. 
En otras ocasiones, el trabajo propuesto a un grupo de alumnos de $1^{\circ}$ de ESO ha consistido en que cada uno de ellos estudiara un objeto del patrimonio para luego, a modo de guía, explicárselo a otros compañeros o a sus padres. Un vídeo de esta actividad puede verse en el enlace:

http://ceimes.cchs.csic.es/aula_actual/historia_isabel_catolica

\section{PROYECTOS CON LOS ALUMNOS DE $4^{\circ}$ DE ESO}

Con los alumnos de $4^{\circ}$ de ESO, nos planteamos proyectos más complejos porque su nivel de madurez y conocimientos es obviamente mayor que el de los alumnos de $1^{\circ}$. Los objetivos inmediatos de los proyectos son dos: 1) que profundicen en el estudio y valoración del patrimonio del InstitutoEscuela, y 2) que desarrollen las capacidades relacionadas con el trabajo en equipo, la investigación básica a partir de fuentes históricas, el uso de las tecnologías de la información para la búsqueda de datos y la exposición de resultados, y la presentación clara y ordenada de trabajos escritos.

Los departamentos que han participado desde el primer momento en estos proyectos han sido los de Geografía e Historia, Biología y Geología, Tecnología (Informática) y, de manera puntual, otros como los de Educación Plástica y Visual y Educación Física. En sus trabajos, los alumnos, dirigidos por profesores de los citados departamentos, tratan con carácter monográfíco a lo largo del curso aspectos relevantes y concretos del Instituto-Escuela a partir de las fuentes patrimoniales. Todos esos trabajos se han ido publicando anualmente, coincidiendo con el fin del curso, en una revista de difusión interna del instituto, y se pueden ver en el enlace:

https://www.iesisabellacatolica.es/index.php/actividades/recuperacion-del-patrimonio

Las fuentes patrimoniales utilizadas hasta ahora por los alumnos de $4^{\circ}$ de ESO en los trabajos han sido:

1. Los expedientes de los alumnos del Instituto-Escuela ${ }^{4}$ : fueron el punto de partida del proyecto didáctico Alumnos del Instituto-Escuela: Trayectorias, realizado en el curso 20142015, en el que participaron los departamentos de Geografía e Historia y Tecnología (Informática). Con el estudio de una selección de expedientes de este fondo documental, nuestros alumnos de $4^{\circ}$ descubrieron distintos aspectos del sistema de enseñanza del Instituto-Escuela: cuántas asignaturas había en cada curso del Bachillerato, cuál era la opcionalidad en los dos últimos cursos, qué idiomas estudiaban los alumnos, cómo se los calificaba, las comunicaciones a los padres, etc. Después de analizar sus expedientes académicos, buscaron datos biográficos en Internet para reconstruir sus respectivas trayectorias profesionales. Así pudieron comprobar, de paso, cómo aquéllos habían vivido las etapas y acontecimientos históricos que ellos estudiaban en $4^{\circ}$ de la ESO en Historia: dependiendo de sus edades, los alumnos del Instituto-Escuela estudiaron durante el último periodo del reinado de Alfonso XIII, la Dictadura de Primo de Rivera y la Segunda República, vivieron la Guerra Civil y desarrollaron su vida profesional bajo el franquismo o en el exilio. En la clase de Informática, los alumnos de $4^{\circ}$ hicieron la búsqueda de datos en Internet y la exposición final del trabajo en forma de presentación.

4 El Instituto Isabel la Católica conserva los expedientes de 900 alumnos que estudiaron en el InstitutoEscuela entre 1918 y 1936. 
2. Los cuadernos de clase: fueron una herramienta fundamental para el aprendizaje en el Instituto-Escuela, hasta tal punto que, como ya hemos comentado, en los primeros cursos del Bachillerato sustituyeron a los libros de texto. Los utilizamos en el curso 2011-2012 para el proyecto didáctico La enseñanza de la Geografía y de la Geología en el Instituto-Escuela, dirigido por los departamentos de Geografía e Historia y Biología y Geología. Para realizar esta actividad, cedimos a nuestros alumnos una colección de cuadernos digitalizados de Geografía del antiguo alumno del Instituto-Escuela Francisco Javier Cabañas Rodríguez. Nos inclinamos por los cuadernos de Geografía porque esta disciplina se renovó a principios del siglo XX, cuando se convirtió en la ciencia que estudia los paisajes naturales modificados por el hombre. En el Instituto-Escuela enseñaron dos de los renovadores de la Geografía española, Juan Dantín Cereceda (de 1919 a 1921) y Manuel de Terán (de 1923 a 1930, como profesor aspirante, y de 1931 a 1936 como catedrático), por lo que en los cuadernos de clase de sus alumnos ya se aprecia ese nuevo enfoque disciplinar. Al analizar los cuadernos de Geografía de España, de Europa y de otros continentes en sus aspectos humano y económico, nuestros alumnos constataron los cambios trascendentales que se han producido en el mundo desde principios del siglo XX, la fecha de los cuadernos, hasta comienzos del siglo XXI, cuando ellos los estudian. Por otra parte, pudieron comparar los cuadernos de clase de los alumnos del Instituto-Escuela y los suyos propios en cuanto a contenidos, ejercicios, ilustraciones, correcciones de los profesores, calificaciones, etc. El resultado de la comparación fue muy enriquecedor para nuestros alumnos. Un artículo específico sobre este proyecto se puede ver en el enlace:

http://revista.muesca.es/documentos/cabas9/La-ensenanza-de-la-Geografia.pdf

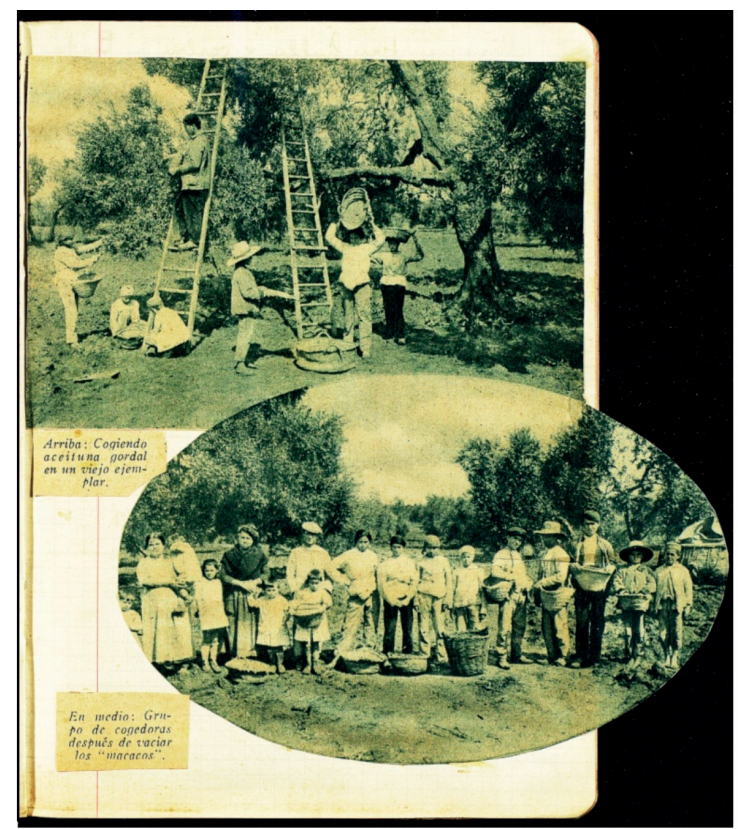

Figura 6. Cuaderno de Geografía del alumno del Instituto-Escuela Javier Cabañas en $2^{\circ}$ de Bachillerato. Curso 19301931. Fondo Javier Cabañas. 
3. Las Memorias del Instituto-Escuela: en ellas basamos dos proyectos didácticos, Las alumnas del Instituto-Escuela y La educación física y artística en el Instituto-Escuela. En el primero, realizado en el curso 2009-2010, intervinieron los departamentos de Geografía e Historia y Tecnología, Los alumnos de $4^{\circ}$, partiendo de los datos de las Memorias, analizaron los porcentajes de alumnas y alumnos del Instituto-Escuela, que fue de un $45 \%$ y $55 \%$ respectivamente. Con ayuda de los datos proporcionados on line por el Instituto Nacional de Estadística, consultaron después cuáles eran los porcentajes en los otros Instituto oficiales madrileños del primer tercio del siglo XX y, comparándolos, llegaron a la conclusión de que el número de alumnas que estudiaban en el Instituto-Escuela era muy superior al de los referidos institutos, donde su presencia apenas llegaba al 20\%. Estos datos sirvieron para reflexionar sobre las causas de la escasa presencia femenina en la enseñanza de esa época, salvo en el Instituto-Escuela. El trabajo fue completado por nuestros alumnos de $4^{\circ}$ con la búsqueda de datos biográficos de algunas de sus alumnas que, siendo ya adultas, tuvieron una trayectoria profesional relevante.

El segundo proyecto, La educación física en el Instituto-Escuela, lo desarrollamos a lo largo del curso 2016-2017. Los datos de las Memorias nos sirvieron en esa ocasión para obtener información acerca de su plan de estudios. En él consta el número de horas dedicadas a cada materia $\mathrm{y}$, en concreto, a su formación física. Al comparar el número de horas que el plan de estudios del Instituto-Escuela dedicaba a los Juegos y deportes, con los otros planes de estudios vigentes (Plan Bugallal de 1903, Plan Calleja de 1926, y Plan Villalobos de 1934), nuestros alumnos pudieron constatar, en primer lugar, la importancia educativa que el Instituto-Escuela le dio a los Juegos y deportes, fuente de disciplina, sociabilidad y trabajo en equipo, según María de Maeztu; y, en segundo lugar, el papel de pioneros deportivos desempeñado por los alumnos y las alumnas del Instituto-Escuela. Nuestro proyecto se centró esta vez en aquellas alumnas del Instituto-Escuela que fueron pioneras del deporte femenino español, y fue realizado por los departamentos de Geografía e Historia, Tecnología y Educación Física. Las alumnas del Instituto-Escuela, por haber hecho estudios superiores en un porcentaje mayor que otras de otros institutos, fueron mayoría entre las tituladas universitarias de la época y las primeras en incorporarse a profesiones y actividades reservadas tradicionalmente a los hombres, incluidos los deportes. Después, el trabajo de nuestros alumnos de $4^{\circ}$ se centró en las biografías y en las trayectorias profesionales de dos profesoras de deportes del Instituto-Escuela y de dos alumnas que fueron pioneras en sus especialidades deportivas. Así, nuestros alumnos pudieron comprobar cómo les afectó la Guerra Civil y cómo vivieron bajo el franquismo situaciones que, en algún caso, conllevaron la represión y el exilio.

4. El material científico de los laboratorios de Biología y Geología: Lo escogimos para desarrollar dos proyectos con alumnos de $4^{\circ}$, Los profesores de Ciencias que se formaron en el Instituto-Escuela y La enseñanza de las Ciencias Naturales en el Instituto-Escuela. Francisco Bernis en su centenario. En ambos proyectos participaron los departamentos de Biología y Geología, Geografía e Historia, y Tecnología. En el primer proyecto, realizado a lo largo del curso 2010-2011, nos centramos en la importancia que tuvo la formación del profesorado en el Instituto-Escuela. Los materiales científicos que utilizaron los profesores de Ciencias Naturales, y que conservamos, les permitieron a nuestros alumnos conocer su método de enseñanza, el cual se basaba, por una parte, en la presencia de los objetos a estudiar para hacer la enseñanza más comprensiva (observando 
de manera directa las cosas a través de láminas, placas de cristal, microfotografías, modelos anatómicos y florales o animales naturalizados); y por otra parte, en la experimentación que hacían los propios alumnos en los laboratorios destinados a ese fin. Los instrumentos y enseres conservados (microscopios, micrótomo, preparaciones realizadas por los alumnos, etc.) constituyen una muestra muy clara de esa enseñanza activa y experimental que preconizaba el Instituto-Escuela.

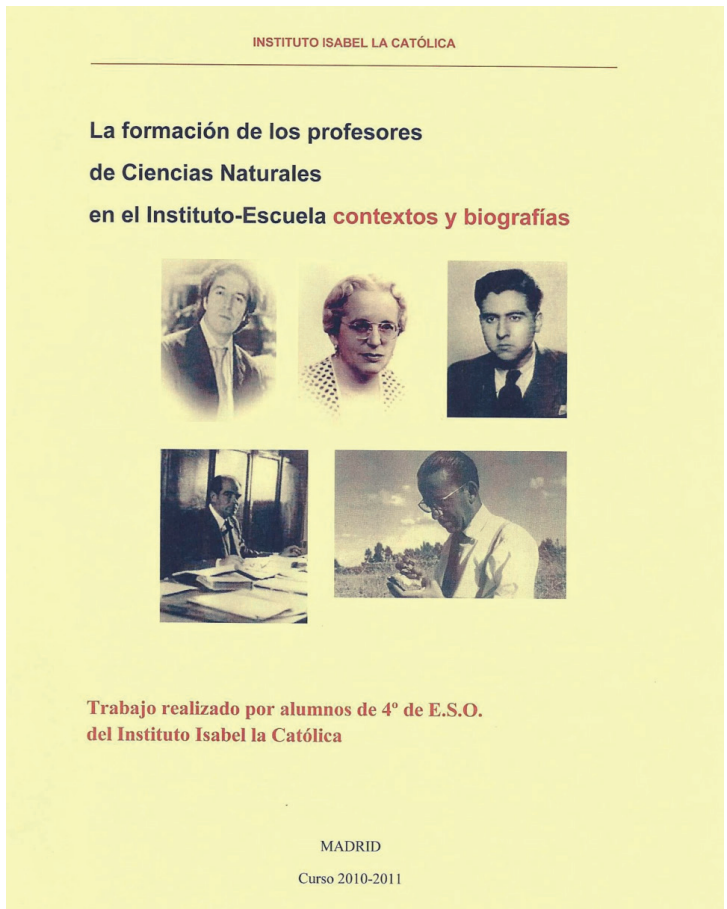

Figura 7. Portada de la revista Los profesores de Ciencias Naturales que se formaron en el Instituto-Escuela.

Entre los profesores aspirantes de Ciencias Naturales formados pedagógicamente en el Instituto-Escuela, nos centramos en la figura de Vicente Sos Baynat (1895-1992), del que hicimos un estudio conjunto con los alumnos y profesores de los Institutos Albarregas (Mérida) y Sos Baynat (Castellón). Cuando éstos visitaron nuestro instituto para compartir conocimientos sobre Sos Baynat, nuestros alumnos de $4^{\circ}$ les mostraron el patrimonio científico que habría utilizado este profesor en el Instituto-Escuela. Después, con la relación de los profesores de Ciencias Naturales formados en él, extraída de las Memorias, hicieron búsquedas informáticas y elaboraron gráficos a partir de los datos recabados relacionados con las instituciones científicas donde se perfeccionaron en su especialidad, el número de los becados en el extranjero por la JAE, los que se exiliaron al acabar la Guerra Civil, y la proporción de hombres y mujeres que hubo entre ellos. Finalmente trabajaron en sus biografías y trayectorias profesionales. 
El otro proyecto, La enseñanza de las Ciencias Naturales en el Instituto-Escuela. Francisco Bernis en su centenario, fue realizado en el curso 2015-2016. Los alumnos empezaron estudiando, como en el proyecto anterior, el material científico de los laboratorios para entender la pedagogía aplicada en la enseñanza de las Ciencias Naturales, y luego pasaron a ocuparse de la figura de Francisco Bernis Madrazo (1916-2003). Bernis estudió el Bachillerato en el Instituto-Escuela entre 1927 y 1933, y años después llegó a ser catedrático de instituto y catedrático de Vertebrados en la Universidad de Madrid, Jefe de Vertebrados del Museo Nacional de Ciencias Naturales y un gran ornitólogo. Con nuestro proyecto queríamos conmemorar el centenario de su nacimiento y comprobar cómo posiblemente las enseñanzas recibidas en el Instituto-Escuela pudieron influir en su vocación ornitológica y conservacionista. Este trabajo de los alumnos, además de editarse en una revista, se completó con carteles y vídeos realizados en clase de Informática, y con una exposición en el instituto a cuya inauguración asistieron los hijos de Francisco Bernis Madrazo.

5. La Biblioteca histórica: Con su fondo bibliográfico hemos realizado dos proyectos, ambos durante el curso 2013-2014. El primero de ellos, Emilio Guinea y la flora de Guinea, fue sobre Emilio Guinea, profesor aspirante de Ciencias Naturales en el Instituto-Escuela, que después fue conservador y director del Real Jardín Botánico (RJB), así como un eminente científico estudioso de la flora de Guinea Ecuatorial. El elemento patrimonial que nos sirvió de referencia fue el libro de Emilio Guinea Compendio de la flora de Guinea Ecuatorial, conservado en nuestra biblioteca histórica, en el que aparecen reproducidas 10 acuarelas del propio autor. El proyecto, en el que participaron excepcionalmente alumnos de $3^{\circ}$ de ESO, fue dirigido por los departamentos de Educación Plástica y Visual y Biología y Geología, y contó con el apoyo del RJB, que luego visitaron para conocer la labor investigadora de Emilio Guinea. Después en la clase de Educación Plástica y Visual practicaron con la técnica de la acuarela reproduciendo las de Emilio Guinea, y en la clase de Ciencias Naturales estudiaron la flora tropical.

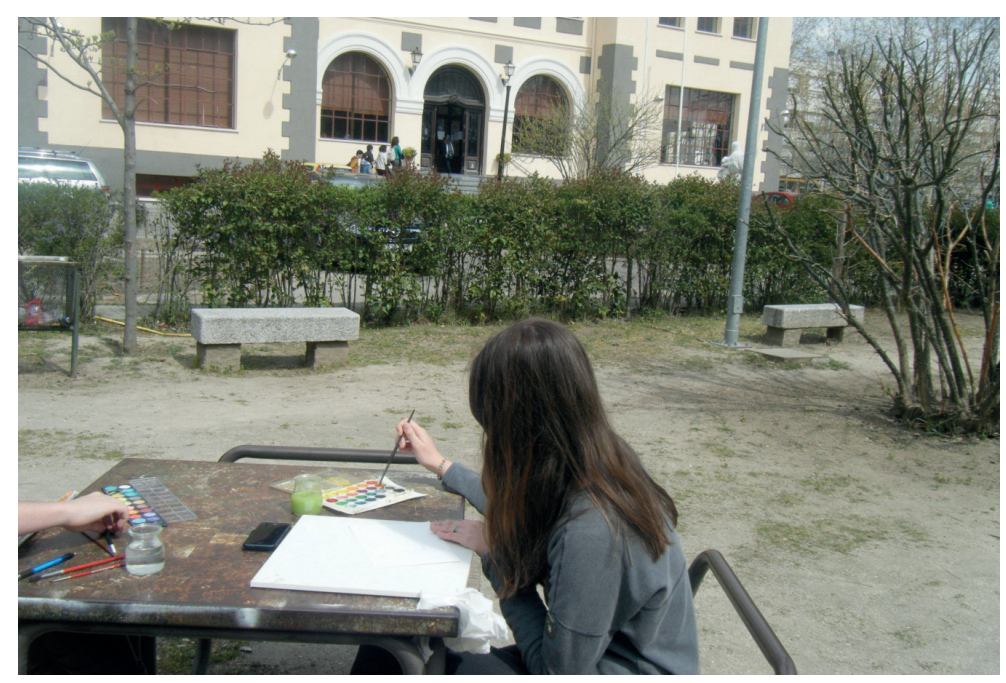

Figura 8. Alumna pintando una acuarela frente a la fachada del Instituto Isabel la Católica. 
En el mismo curso, con los alumnos de $4^{\circ}$ de ESO, utilizamos el Atlas Universel de Robert de Vaugondy, publicado en París en 1810, como recurso didáctico para otro proyecto interdisciplinar en el que participaron los departamentos de Geografía e Historia y Tecnología. Esta obra no procede de la biblioteca del Instituto-Escuela sino de la donación que hizo Enrique Canito Barreda, catedrático de francés en el Instituto Isabel la Católica entre 1945 y $1972^{5}$. El atlas, del que sólo se conservan cinco ejemplares en España, fue restaurado en 2013 y sobre su proceso de restauración montamos una exposición que fue el punto de partida para la actividad que realizamos después con los alumnos. En clase de Historia, trabajaron con una selección de mapas digitalizados del atlas que les sirvieron como mapas históricos para ver cuál era la distribución territorial del mundo en 1810. A partir de ahí estudiaron los cambios producidos en todo el siglo XIX, los relacionaron con los hechos históricos estudiados en la materia de Historia de $4^{\circ}$ de ESO, y analizaron la cartografía de la época del atlas. Terminaron su trabajo en clase de Informática con una presentación en Prezi.

6. Las maquetas: El Instituto Isabel la Católica posee cuatro maquetas didácticas cedidas en depósito por el Museo Nacional de Ciencias Naturales. El motivo de esta cesión es que fueron proyectadas por el catedrático de Ciencias Naturales formado en el Instituto-Escuela Carlos Vidal Box (1906-1972), cuando era inspector de enseñanza media en los años 40 del siglo pasado. De estas maquetas, elegimos la de Toledo para realizar en el curso 2012-2013 el proyecto Recordando las excursiones del Instituto-Escuela: estudio geológico e histórico-artístico de Toledo, en el que participaron los departamentos de Biología y Geología Geografía e Historia, y Tecnología. La actividad nos sirvió para reflexionar sobre el papel de las excursiones en la educación, que tanta importancia tuvo en el Instituto-Escuela, y para comprobar cómo aún hoy, pese a que los alumnos pueden acceder a las imágenes y a la información por muchos más medios, las excursiones siguen siendo un recurso insustituible del aprendizaje porque permiten contemplar determinados objetos de estudio in situ. Para preparar la excursión, en las clases de Ciencias Naturales, los alumnos estudiaron la geología de Toledo; en las de Historia, la evolución histórica y los aspectos más destacados de la geografía urbana de la ciudad, y en las de Tecnología obtuvieron la información geográfica a través de Google Earth y los visores más actualizados, como SIG, InfoIGME y Arcgis.

En el curso 2017-2018, hemos hecho una recopilación de algunos de los referidos proyectos sobre el uso didáctico del patrimonio de nuestro instituto en dos vídeos, uno realizado por la Comunidad de Madrid y otro por la UNED, que se pueden ver en los siguientes enlaces:

\section{https://mediateca.educa.madrid.org/lista/Lfrwfmolfoqqxb3g4 https://canal.uned.es/video/5b338163b1111fe7498b4567}

Desde que descubrimos el patrimonio histórico del Instituto-Escuela depositado en el Instituto Isabel la Católica, nos hemos dedicado a recuperarlo, estudiarlo, catalogarlo, restaurarlo $\mathrm{y}$, en la medida de lo posible, difundirlo para que la sociedad de nuestro tiempo pueda acceder al conocimiento de una experiencia educativa singular en la historia de la educación española, y precursora de los planes de educación de nuestra etapa democrática. Que, por otra parte, los profesores

Enrique Canito simultaneó su labor docente con la de director de la Revista literaria Ínsula y de la librería del mismo nombre. 
y alumnos del instituto lo estén utilizado como recurso didáctico en actividades interdisciplinares, ha permitido ensanchar y mantener viva la memoria del Instituto-Escuela en el ámbito de nuestra comunidad escolar.

Al cumplirse el centenario de la creación del Instituto-Escuela, la exposición que con este motivo se va a celebrar en Madrid, en el Museo Nacional de Ciencias Naturales, del 30 de octubre de este año hasta el 10 de enero de 2019, queremos que contribuya a que su experiencia educativa sea conocida y valorada por un público mucho más amplio.

\section{REFERENCIAS}

Canes Garrido, F. (2001). El debate sobre los libros de texto de Secundaria en España (1875-1931). Revista Complutense de Educación, Madrid, vol.12, nº 1, pp. 357-395.

Casado de Otaola, S. (2012). Celso Arévalo, catedrático del Instituto Cardenal Cisneros y la modernización de la enseñanza de las Ciencias Naturales en el primer tercio del siglo XX. En L. López-Ocón, S. Aragón y M. Pedrazuela (Eds.). Aulas con Memoria. Ciencia, educación y patrimonio en los Instituto históricos de Madrid (1837-1936). Madrid: CEIMES. Doce Calles. pp. 172-188

Díaz de Laguardia, E. (1988). Evolución y desarrollo de la enseñanza media en España de 1875 a 1930. Un conflicto político-pedagógico. Madrid: Ministerio de Educación y Ciencia. C.I.D.E.

Junta para Ampliación de Estudios (1925). Un ensayo pedagógico. El Instituto Escuela de Segunda Enseñanza de Madrid (Organización, Métodos, Resultados). Madrid.

Junta para Ampliación de Estudios, Memorias bianuales: 1920, 1922, 1925, 1927, 1929, 1930, 1933, 1935. Madrid

López-Ocón, L. (2014). Reflexiones sobre la modernidad en las aulas de bachillerato en el primer tercio del siglo XX. En L. López-Ocón (Ed.), Aulas Modernas. Nuevas perspectivas sobre las reformas de la enseñanza secundaria en la época de la JAE (1907-19039) Madrid: Universidad Carlos III, pp. 9-46.

Martínez Alfaro, E. (2009). Un laboratorio pedagógico de la Junta para Ampliación de Estudios. El Instituto-Escuela Sección Retiro de Madrid. Madrid: Biblioteca Nueva.

Martínez Alfaro, E. y Masip Hidalgo, C. (2012a). La recuperación del patrimonio del Instituto-Escuela en el Instituto Isabel la Católica. En L. López-Ocón, S. Aragón y M. Pedrazuela (Eds.), Aulas con Memoria. Ciencia, educación y patrimonio en los Instituto históricos de Madrid (1837-1936). Madrid: CEIMES. Doce Calles, pp. 135-149.

Martínez Alfaro, E. y Masip Hidalgo, C. (2012b). Cuadernos y trabajos escolares: una práctica innovadora en el Instituto-Escuela. En L. López-Ocón, S. Aragón y M. Pedrazuela (Eds.), Aulas con Memoria. Ciencia, educación y patrimonio en los Instituto históricos de Madrid (1837-1936). Madrid: CEIMES. Doce Calles, pp. 225-241.

Melcon Beltrán, J. (2000). Currículo escolar y lecciones de cosas. En A. Tiana (Dir.), El libro escolar, reflejo de intenciones políticas e influencias pedagógicas. Madrid: UNED, pp. 135-160.

Palacios Bañuelos, L. (1988). Instituto-Escuela. Historia de una renovación educativa. Madrid: Ministerio de Educación y Ciencia. 
Ruiz Berrio, J. (2012). El Instituto-Escuela. En J. García Velasco y A. Morales Moya (ed.), La Institución Libre de Enseñanza y Francisco Giner de los Ríos: Nuevas perspectivas. T.2. La Institución Libre de Enseñanza y la cultura española. Madrid: Fundación Francisco Giner de los Ríos y Acción Cultural Española, pp. 578-593.

Utande, M. (1964). Planes de estudio de Enseñanza Media. Madrid: Ministerio de Educación Nacional. 